\section{To compare argon \\ laser peripheral \\ iridoplasty (ALPI) \\ against systemic \\ medications in \\ treatment of acute \\ primary angle- \\ closure: mid-term \\ results}

JSM Lai'1,2, CCY Tham², JKH Chua², ASY Poon², JCH Chan'2, SW Lam² and DSC Lam²

\begin{abstract}
Purpose To compare the clinical outcome of argon laser peripheral iridoplasty (ALPI) against systemic medications in treatment of acute primary angle-closure (APAC).

Methods Consecutive patients with APAC were recruited and randomized to receive one of two treatment options: immediate ALPI or systemic acetazolamide \pm mannitol. All eyes were followed up for at least 6 months after laser iridotomy. Main outcome measures were intraocular pressure (IOP) and requirement for glaucoma medications.
\end{abstract}

Results A total of 41 eyes (39 patients) were randomized into the ALPI group, and 38 eyes ( 32 patients) into the medical treatment group. There were no significant differences between the two groups in sex, age, presenting IOP, and duration of attack. Mean follow-up duration \pm SD was $15.7 \pm 5.8$ months. There were no significant differences between the two groups in mean final IOP and requirement for glaucoma medications.

Conclusions There were no statistically significant differences in mean IOP and requirement for glaucoma drugs between APAC eyes treated with ALPI and systemic medications.

Eye (2006) 20, 309-314. doi:10.1038/sj.eye.6701867; published online 8 July 2005

Keywords: argon laser peripheral iridoplasty; acute primary angle-closure; medical treatment
Introduction

In eyes with acute primary angle-closure (APAC), the cornea is often so oedematous that the definitive treatment of laser peripheral iridotomy cannot be immediately and safely performed. Under such circumstances, it is usual practice to employ intraocular pressure (IOP)-lowering medications first to reduce the IOP and to allow the cornea to clear up, before attempting laser peripheral iridotomy.

Aung et $\mathrm{al}^{1}$ reported that $58.1 \%$ of Asian eyes with APAC, treated with medications in the conventional manner followed by laser iridotomy, subsequently developed increase in IOP. This finding raised the suspicion that the conventional approach of administering topical and systemic IOP-lowering medications, followed by laser peripheral iridotomy, in APAC may not be the ideal treatment modality, especially in Asian eyes when long-term outcome is taken into consideration.

Argon laser peripheral iridoplasty (ALPI) has been shown to dramatically lower the IOP, with opening up of the closed angle, in patients with APAC. ${ }^{2-7}$ However, its long-term effect on the clinical course is not known. Lai et $a l^{8}$ reported a group of Chinese patients with APAC successfully treated with immediate laser peripheral iridoplasty, followed by peripheral iridotomy, and found that only $30 \%$ of the eyes developed into CACG over a mean follow-up of 33 months. However, this study was uncontrolled.
${ }^{1}$ United Christian Hospital, Kowloon, Hong Kong, People's Republic of China

${ }^{2}$ Department of Ophthalmology and Visual Sciences, The Chinese University of Hong Kong, Hong Kong, People's Republic of China

Correspondence: JSM Lai, Department of Ophthalmology, United Christian Hospital, 130 Hip Wo Street, Kwun Tong, Kowloon, Hong Kong Tel: + 85223513 3411; Fax: + 852235135626 E-mail: laism@ ha.org.hk

Received: 7 October 2004 Accepted: 15 February 2005 published online: 8 July 2005

Financial support: The work described in this paper was substantially supported by a grant from the Research Grants Council of the Hong Kong Special Administrative Region. (Project no. CUHK4090/01M).

Proprietary interest: Nil. 
In this randomized controlled trial, we directly compared the mid-term clinical outcomes of immediate ALPI against systemic medications as the first-line preiridotomy therapy in APAC.

\section{Materials and methods}

The design of this multicentred prospective randomized controlled trial was described in detail previously. ${ }^{6}$

The study has fully complied with the tenets of the Declaration of Helsinki. The research was approved by the institutional human experimentation committee. Consecutive patients with their first presentation of APAC at the United Christian Hospital and Prince of Wales Hospital were recruited into the study. Informed consent was obtained from the subjects after explanation of the nature and possible consequences of the study. Recruited patients were then randomized into one of two treatment groups (ALPI group and medical treatment group), using a random number table. Neither the front line ophthalmologists who diagnosed the APAC, nor the patients, were aware of which treatment group to join at the point of recruitment. For patients with bilateral attack, both eyes were randomized together for the same treatment, that is, either bilateral ALPI or medical treatment only. Exclusion criteria included: (1) IOP less than $40 \mathrm{mmHg}$ on presentation, (2) pupillary block clinically not the main mechanism of angle closure, (3) ophthalmic treatment received prior to presentation, (4) cornea clear enough for immediate laser peripheral iridotomy, (5) corneal opacity obstructing laser access to more than one quadrant of peripheral iris, and (6) singleeyed patients.

For all randomized patients, IOP measurement by Goldmann applanation tonometry, documentation of visual acuity, pupillary reaction and size, and gonioscopy were performed. Gonioscopy was performed using the single-mirror gonio diagnostic lens (Ocular Instruments Inc., Washington, USA) at the slit lamp under topical anaesthesia. Grading of the angle width was in accordance with the Schaffer system. Indentation gonioscopy using a Posner Diagnostic and Surgical Gonioprism (Ocular Instruments, Inc., Bellevue, USA) was performed when a closed angle was encountered, to distinguish between appositional and synechial closures. Each APAC eye was treated with one drop of topical pilocarpine $4 \%$, and one drop of topical timolol maleate $(0.5 \%)$, irrespective of treatment group.

For the ALPI group, ALPI was performed under topical anaesthesia, as previously described. ${ }^{6}$ The laser was initially set at an energy level of $200 \mathrm{~mW}$, and titrated according to response. The duration of each laser pulse was $0.5 \mathrm{~s}$, with a spot size of $500 \mu \mathrm{m}$. The laser beam was focused onto the peripheral iris as close to the limbus as possible with the Abraham Iridectomy Laser Lens (Ocular Instruments, Inc., Bellevue, USA). All four quadrants $\left(360^{\circ}\right)$ were treated whenever possible. The end point was reached when localized iris contraction at the treated area became visible. The treated eye was maintained on topical pilocarpine (1\%) four times a day until peripheral laser iridotomy.

For the medical treatment group, each patient was given $500 \mathrm{mg}$ of intravenous acetazolamide, followed by oral acetazolamide $250 \mathrm{mg}$ four times a day, and oral potassium supplement $600 \mathrm{mg}$ twice a day, until IOP normalized. If IOP was greater than $60 \mathrm{mmHg}$ at presentation, $200 \mathrm{ml}$ of $20 \%$ mannitol would also be administered intravenously over $1 \mathrm{~h}$. In patients with bilateral attack, intravenous mannitol would be given if IOP of either eye was higher than $60 \mathrm{mmHg}$ at presentation. The treated eye was maintained on topical pilocarpine (1\%) four times a day until peripheral laser iridotomy.

For both groups of patients, IOP was measured by Goldmann applanation tonometry at $15 \mathrm{~min}, 30 \mathrm{~min}, 1 \mathrm{~h}$, $2 \mathrm{~h}$, and 1 day after commencement of treatment. Gonioscopy was repeated 1 day after presentation.

Topical steroid (Pred Forte 1\%) four times per day was prescribed as required, and timolol would be continued if IOP remained above $20 \mathrm{mmHg}$. Laser peripheral iridotomy for the APAC eye was performed as soon as sufficient corneal clarity had returned. The fellow eye was treated with topical pilocarpine (1\%) four times a day until peripheral laser iridotomy, if gonioscopy revealed an occludable angle.

All the patients were followed up at 1 month after the initial attack, and then at 3-monthly intervals. Additional clinic visits were scheduled if clinically indicated. A special data sheet was designed to collect all the follow-up data in the two treatment groups.

Primary outcome measures were IOP (the mean of two Goldmann applanation tonometry readings taken at the same clinic visit) and the number of glaucoma medications. Secondary outcome measures included visual acuity, corneal clarity, pupil reaction and diameter, iris appearance, lens clarity, indentation gonioscopy, vertical cup-to-disc ratio, automated perimetry results, and central endothelial cell count (measured by a masked technician using the Konan Specular Microscope (Noncon Robo SP 6000, Japan). Corneal clarity was simply classified as either clinically clear, or oedematous. Pupil reaction was classified as either present, or none. Peripheral anterior synechiae (PAS) was defined as adhesion of the peripheral iris to the drainage angle, which obstructed the pigmented part of the trabecular meshwork from direct aqueous access and could not be separated by indentation gonioscopy. The treatment groups were not 
revealed to the clinicians reviewing the patients at follow-up appointments.

Only patients followed up for more than 6 months after presentation were included in this mid-term review. Patients who had intraocular surgery not intended for IOP control performed within 6 months after the acute attack were excluded from the analysis. For patients who had intraocular surgery performed more than 6 months from the acute attack, all the outcome measures were taken at the point immediately prior to the surgery.

During follow-up, medications were started in the following sequence if the IOP was above $21 \mathrm{mmHg}$ on more than two clinic visits: topical beta-blocker, topical pilocarpine, topical carbonic anhydrase inhibitor, topical prostaglandin analog, systemic acetazolamide. If there was no significant reduction in IOP $(<2 \mathrm{mmHg})$ after adding one drug, that drug would be stopped and another drug further along the sequence started. If there was significant reduction in IOP $(>2 \mathrm{mmHg})$ but the reduction was not large enough to reach below $21 \mathrm{mmHg}$ after adding one drug, that drug would be maintained and the next drug along the sequence would be added.

Any statistical significance in difference in a continuous outcome parameter between the two treatment groups was tested using the Student's $t$-test. $\chi^{2}$ test was used to compare the female-to-male ratios, the right eye-to-left eye ratios, the number of eyes requiring medical treatment to control IOP, the number of eyes with PAS of various extents, and the number of eyes with nonreactive pupil, between the two groups. A $P$-value of $<0.05$ was taken as statistically significant.

\section{Results}

From August 2000 to March 2002, a total of 91 APAC eyes of 83 patients were randomized and treated as described above. All had IOP lowered to less than $21 \mathrm{mmHg}$ within $2 \mathrm{~h}$ and all had laser peripheral iridotomy performed within $48 \mathrm{~h}$ from presentation. Of all the 83 patients, 71 of them (79 eyes) fulfilled all the criteria for final analysis. The reasons for exclusion in the 12 patients (12 eyes) excluded from final analysis were presented in Table 1. None of the study eyes required any IOP-lowering surgery within the follow-up period.

Table 2 summarizes the demographic and clinical data at presentation of these 79 eyes. There were no statistically significant differences in the female-to-male ratio $\left(P=0.40, \chi^{2}\right.$ test $)$, the mean age $(P=0.10, t$-test $)$, right eye-to-left eye ratio $\left(P=0.90, \chi^{2}\right.$ test), IOP at presentation $(P=0.07, t$-test), and duration of attack before treatment $(P=0.17, t$-test), between the two treatment groups. In both treatment groups, all

Table 1 Reasons for exclusion in the 12 patients (12 eyes) not included in the final analysis

\begin{tabular}{lcc}
\hline & Immediate ALPI group & Medical treatment group \\
\hline Number of patients (eyes) at recruitment & $44(46)$ & $39(45)$ \\
Number of patients (eyes) in final analysis & $39(41)$ & $32(38)$ \\
Number of patients dying of unrelated disease (\%) & $1(2.3 \%)$ & $0(0 \%)$ \\
Number of patients defaulting follow-up (\%) & $3(6.8 \%)$ & $1(2.6 \%)$ \\
Number of patients having cataract extraction within & $1(2.3 \%)$ & $4(12.5 \%)$ \\
1 year from acute attack (\%) & $0(0 \%)$ & $1(2.6 \%)$ \\
Number of patients having vitrectomy for proliferative & & $1 \%$ diabetic retinopathy within 1 year from acute attack $(\%)$
\end{tabular}

Table 2 Demographics of the 79 APAC eyes

\begin{tabular}{lccc}
\hline & Immediate ALPI & Medical treatment & P-value \\
\hline Number of eyes (patients) with follow-up $>6$ months & $41(39)$ & $38(32)$ & $29: 3$ \\
Female $:$ male ratio & $32: 7$ & $66.5 \pm 8.5$ & $0.40^{*}$ \\
Mean age \pm SD (years) & $70.0 \pm 10.5$ & $18: 20$ & $0.10^{* *}$ \\
OD: OS ratio & $20: 21$ & LP to 0.5 & $0.90^{*}$ \\
Range of visual acuity during acute attack & LP to 0.6 & $57.1 \pm 9.2$ (Range, 40-74) & $0.07^{* *}$ \\
Mean presenting IOP \pm SD (mmHg) & $61.2 \pm 10.6$ (Range, 40-79) & $29.7 \pm 23.8$ (Range, 4-96) & $0.17^{* *}$ \\
Mean duration of attack \pm SD (h) & $41.6 \pm 47.6$ (Range, 2-168) & $0.26^{* *}$ \\
Mean duration of follow-up \pm SD (months) & $15.0 \pm 6.0$ (Range, 6-36) & $16.4 \pm 5.6$ (Range, 7-29) \\
\hline
\end{tabular}

$\mathrm{ALPI}=$ argon laser peripheral iridoplasty; $\mathrm{SD}=$ standard deviation; $\mathrm{OD}=$ right eye; $\mathrm{OS}=$ left eye; $\mathrm{LP}=$ light perception only; $\mathrm{IOP}=$ intraocular pressure. ${ }^{*} \chi^{2}$ test.

${ }^{* *} t$-test. 
iridotomies were performed as soon as corneal clarity allowed, and all were performed within $48 \mathrm{~h}$ of presentation. There was no statistically significant difference between the two treatment groups in the duration between presentation and laser iridotomy.

Two patients (two eyes) assigned to the ALPI group failed to have IOP controlled by ALPI alone, and systemic medical therapy was then given. One of these eyes developed into chronic angle-closure glaucoma (CACG), and cataract extraction was performed within 6 months after the acute attack. The other eye had IOP controlled after laser peripheral iridotomy without any medication up to the final follow-up. These two eyes were included in the ALPI group in the analysis.

The mean follow-up duration \pm 1 standard deviation (SD) was $15.0 \pm 6.0$ months in the ALPI group and $16.4 \pm 5.6$ months in the medical group $(P=0.26, t$-test). The overall mean follow-up duration \pm 1 SD was $15.7 \pm 5.8$ months (range, $6-36$ months).

The mean IOP at final follow-up \pm 1 SD was $13.6 \pm 2.7 \mathrm{mmHg}$ in the ALPI group, and $14.7 \pm 4.6 \mathrm{mmHg}$ in the medically treated group $(P=0.21)$. Median IOP (range) was $13 \mathrm{mmHg}(9-21 \mathrm{mmHg})$ in the ALPI group, and $14 \mathrm{mmHg}(8-32 \mathrm{mmHg})$ in the medical group. Eight eyes $(19.5 \%)$ in the ALPI group required medical treatment to control IOP, while 12 eyes (31.6\%) in the medically treated group required glaucoma drugs, although this difference was also statistically insignificant $(P=0.22)$. The mean number of glaucoma medications \pm 1 SD was $0.3 \pm 0.7$ in the ALPI group, and $0.5 \pm 0.8$ in the medical group $(P=0.23)$. In the ALPI group, it took a mean duration of $5.1 \pm 6.4$ months for the IOP to rise beyond $21 \mathrm{mmHg}$ before glaucoma treatment was initiated, while in the medical group, it took a mean duration of $3.1 \pm 4.5$ months $(P=0.41)$.

Based on an analysis of the outcome by eyes, significantly more APAC eyes in the medically treated group had PAS of extent $\geq 90^{\circ}$ than in the ALPI group (nine of 41 eyes, or $22.0 \%$, in ALPI group; 17 of 38 eyes, or $44.7 \%$, in medical group) of $(P=0.03)$. However, it is known that studies utilizing data from both eyes of some subjects will, as a consequence of the correlation in risk factors and natural history of disease between right and left eyes (as is the case in angle-closure), produce falsely precise $P$-values and confidence intervals. Given the fact that the difference in PAS has only just attained

Table 3 Outcome measures in the two treatment groups at final follow-up

\begin{tabular}{|c|c|c|c|}
\hline & $\begin{array}{c}\text { Immediate } \\
\operatorname{ALPI}(\mathrm{n}=41)\end{array}$ & $\begin{array}{l}\text { Medical } \\
\text { treatment }(\mathrm{n}=38)\end{array}$ & P-value \\
\hline Mean IOP \pm SD $(\mathrm{mmHg})$ & $13.6 \pm 2.7$ & $14.7 \pm 4.6$ & $0.21^{*}$ \\
\hline Median IOP (range) $(\mathrm{mmHg})$ & $13(9-21)$ & $14(8-32)$ & \\
\hline $\begin{array}{l}\text { Number of eyes requiring medical treatment to control IOP } \\
\text { (percentage of total number of eyes in that group) }\end{array}$ & $8(19.5 \%)$ & $12(31.6 \%)$ & $0.22 * *$ \\
\hline Mean number of medication \pm SD & $0.3 \pm 0.7^{\mathrm{a}}$ & $0.5 \pm 0.8^{\mathrm{b}}$ & $0.23^{*}$ \\
\hline $\begin{array}{l}\text { Mean onset time of ocular hypertension } \pm \text { SD } \\
\text { (months after treatment) }\end{array}$ & $5.1 \pm 6.4$ & $3.1 \pm 4.5$ & $0.41^{*}$ \\
\hline Range of visual acuity & $6 / 120-6 / 6$ & $6 / 120-6 / 6$ & \\
\hline Number of eyes with PAS $\geq 90^{\circ}$ (percentage of total number of eyes in that group) & $9(22.0 \%)$ & $17(44.7 \%)$ & $0.03^{* *}$ \\
\hline Number of eyes with PAS $\geq 180^{\circ}$ (percentage of total number of eyes in that group) & $4(9.8 \%)$ & $8(21.1 \%)$ & $0.16^{* *}$ \\
\hline Number of eyes with PAS $\geq 270^{\circ}$ (percentage of total number of eyes in that group) & $1(2.4 \%)$ & $3(7.9 \%)$ & $0.27^{* *}$ \\
\hline \multirow{2}{*}{$\begin{array}{l}\text { When only one eye of bilateral cases randomly selected for } \\
\text { analysis Number of eyes with PAS } \geq 90^{\circ} \\
\text { (percentage of total number of eyes in that group) }\end{array}$} & nine of 39 eyes & 14 of 32 eyes & $0.06^{* *}$ \\
\hline & $(23.1 \%)$ & $(43.8 \%)$ & \\
\hline Mean cup-to-disc ratio $\pm S D$ & $0.5 \pm 0.2$ & $0.5 \pm 0.2$ & $0.49^{*}$ \\
\hline Median cup-to-disc ratio (range) & $0.4(0.2-0.9)$ & $0.3(0.2-0.9)$ & \\
\hline Mean pupil size $\pm S D(\mathrm{~mm})$ & $3.4 \pm 1.1$ & $2.8 \pm 1.1$ & $0.02^{*}$ \\
\hline $\begin{array}{l}\text { Number of eyes with nonreactive pupil } \\
\text { (percentage of total number of eyes in that group) }\end{array}$ & $13(31.7 \%)$ & $8(21.1 \%)$ & $0.18^{* *}$ \\
\hline Mean endothelial cell count \pm SD $\left(\mathrm{mm}^{-2}\right)$ & $2305.1 \pm 469.7$ & $2449.3 \pm 490.1$ & $0.20^{*}$ \\
\hline
\end{tabular}

$\mathrm{ALPI}=$ argon laser peripheral iridoplasty; $\mathrm{SD}=$ standard deviation; $\mathrm{IOP}=$ intraocular pressure; $\mathrm{PAS}=$ peripheral anterior synechiae

${ }^{a}$ No eyes in the ALPI-treated group required systemic medications to control IOP.

${ }^{b}$ One eye in the medically-treated group required systemic acetazolamide to control IOP.

${ }^{*} t$-test.

** $\chi^{2}$ test. 
'significance' ( $P=0.03$ ), we proceeded to analyse this outcome by patient. We randomly selected one eye of the bilateral cases to be excluded from the analysis. After analysis of the 39 eyes (39 patients) of ALPI group and the 32 eyes ( 32 patients) of the medical group, the difference in proportion of cases with PAS greater than $90^{\circ}$ (nine of 39 patients, or $23.1 \%$ in ALPI group; 14 of 32 patients, or $43.8 \%$ in medical group) was no longer statistically significant $(P=0.06)$.

Table 3 summarizes these primary outcome measures, and also the secondary outcome measures, in the two treatment groups.

The corneas were clinically clear in all eyes at the final follow-up visit.

Two eyes in the ALPI group had decrease in visual acuity due to cataract. Another two eyes had visual acuity remaining at 6/120 and 6/60 due to amblyopia and cataract, respectively. One eye in the medical group had decrease in visual acuity due to ischaemic optic neuropathy. Two eyes had visual acuity remaining at $6 / 30$ and $6 / 120$ due to cataract. There was no obvious difference in range of final visual acuity between the two treatment groups.

The visual acuity test results were not further analysed because many factors, other than the efficacy of the two treatments, were significantly affecting the visual outcome. These factors included the frequency and duration of previous APAC attacks, the density of cataract, and coexisting macular degeneration in some of our study patients.

The mean cup-to-disc ratio $\pm S D$ at the last follow-up was $0.5 \pm 0.2$ in both the ALPI and the medical treatment groups ( $P=0.49, t$-test). Median cup-to-disc ratio (range) was $0.4(0.2-0.9)$ in the ALPI group, and $0.3(0.2-0.9)$ in the medical group. There was no reliable documentation of cup-to-disc ratio in the majority of eyes during the attack, as visualization was affected by corneal oedema.

The visual field test results were not analysed because over $70 \%$ of patients in both groups failed to produce reliable results despite repeated attempts.

None of the patients in either group had reattack of acute angle-closure. No patient required filtering operation during the follow-up period. Three eyes in the ALPI group and one eye in the medical group required cataract extraction at 2,10,12, and 9 months, respectively after the acute attack.

\section{Discussion}

In this study, there were no statistically significant differences between the two treatment groups in mean final IOP, the proportion of eyes requiring glaucoma drugs, and the mean number of glaucoma medications.
There was an observed clinical difference in the proportion of eyes having PAS of extent $\geq 90^{\circ}$ at the final follow-up between the two treatment groups, but statistical significance was only attained when the outcome was analysed by eyes, and not when the analysis was by patient. Larger sample size and/or longer follow-up duration may shed more light on the difference in this outcome between the two treatment groups.

There was an observed clinical difference in the proportion of eyes requiring glaucoma medications to control IOP, with a much lower proportion in the immediate ALPI group (19.5\%) compared with that of the medical treatment group $(31.6 \%)$, although this was statistically insignificant ( $P=0.22, \chi^{2}$ test) (Table 3$)$. These figures are both lower than the $58.1 \%$ reported by Aung et al, ${ }^{1}$ but it should be noted that the study by Aung et al ${ }^{1}$ had a longer mean follow-up of 50.3 months.

There were no statistically significant differences in other clinical outcomes. Based on these results, it appears that immediate ALPI for APAC is at least not associated with a higher subsequent incidence of CACG, as there was a previous concern that the laser might induce additional inflammation in the vicinity of a closed angle in an APAC eye, which could in turn cause more PAS formation.

ALPI reduces IOP more rapidly than systemic medications in the first $2 \mathrm{~h}$ after the initiation of treatment in APAC. ${ }^{6}$ It is believed that ALPI mechanically pulls open the closed drainage angle, and thereby allowing aqueous to escape through the trabecular meshwork. This hypothesis is supported by ultrasound biomicroscopy study. ${ }^{2}$ Since the appositional closure of the drainage angle in a grossly inflamed eye during an acute attack may conceptually predispose to PAS formation, ALPI may, at least in theory, reduce the chance of PAS formation by reducing the duration of appositional angle closure. This may potentially help reduce the chance of subsequent progression to CACG.

After successful laser peripheral iridotomy, plateau iris configuration may still cause angle closure, either acutely or insidiously. ALPI effectively flattens the peripheral iris, and can thus widen the appositionally closed drainage angle in plateau iris syndrome. ${ }^{9}$ It can, at least conceptually, help prevent recurrence of acute angleclosure attack, and also prevent PAS development secondary to an insidious appositional angle closure. These effects are likely to be sustained for years after ALPI. ${ }^{7,9}$ Employing ALPI as the primary treatment for APAC may also deal with any plateau iris configuration at the same time. Whether APAC eyes treated with immediate ALPI has a lower rate of recurrence of the acute attack following successful iridotomy than medically treated cases has yet to be seen in the long 
term. The authors' experience is that during an acute attack, it is often not possible to place ALPI spots as peripherally as one is able to in a cold elective setting, possibly due to anatomical reasons. If this is the case, we may expect immediate ALPI in an APAC eye to be less effective than elective ALPI in a quiet plateau iris eye in eliminating the plateau iris configuration.

ALPI may be associated with potential risks such as direct corneal burn and corneal endothelial cell loss. However, the endothelial cell count did not show a significant difference between the two groups. Apart from the cornea, the laser may also damage the iris. The commonest visible change at the laser application sites on the iris is increased pigmentation. ALPI may also rarely result in iris atrophy. This is believed to be due to direct destruction of the iris vessels by the laser energy. The mean pupil size was significantly larger in the ALPI than the medical treatment group $(P=0.02)$ but the number of nonreactive pupil was similar in both groups $\left(P=0.18, \chi^{2}\right.$ test) (Table 3$)$. The clinical effect of larger pupil size on these patients was not certain. It would have been interesting to see if the larger pupil size, particularly when coupled with the pupil being nonreactive, had resulted in more glare, or discomfort, in the ALPI-treated patients.

In the initial study design, bilateral cases of APAC received the same treatment for both eyes, because analysis would be impossible if one eye received ALPI and the other eye received medical treatment. Although the choice of treatment was randomized, it turned out that the number of bilateral cases in the medical treatment group outnumbered that in the immediate ALPI group by 6-2. This might have introduced bias, as it was not certain if bilateral attack could itself be a risk factor for subsequent poor IOP control. However, analysis of the association between laterality and poor IOP control showed no statistically significant difference between unilateral and bilateral attack $(P=1.00)$ (Fisher's exact test).

The authors do acknowledge that there were inherent limitations in the study design. This is a subject matter in which some of the important outcome measures cannot be properly quantified, and the documentation of which cannot be easily standardized. The amount of peripheral anterior synechiae is a good example. This problem is compounded by the fact that there were multiple investigators reviewing the patients at follow-up appointments at two different hospitals. Furthermore, because laser marks were present on the peripheral iris of APAC eyes treated with ALPI, it was impossible to effectively mask the investigators at follow-ups from the treatment received, and this might have introduced bias.

In conclusion, the results from this randomized controlled study suggest that treatment of APAC with immediate ALPI does not increase the risk of subsequent uncontrolled IOP, compared to treatment with conventional systemic IOP-lowering medications.

\section{References}

1 Aung T, Ang LP, Chan SP, Chew PT. Acute primary angleclosure: long-term intraocular pressure outcome in Asian eyes. Am J Ophthalmol 2001; 131: 7-12.

2 Lam DS, Lai JS, Tham CC. Immediate argon laser peripheral iridoplasty as treatment for acute attack of primary acuteangle closure glaucoma: a preliminary study. Ophthalmology 1998; 105: 2231-2236.

3 Tham CC, Lai JS, Lam DS. Argon laser peripheral iridoplasty in the management of acute primary angleclosure glaucoma without medications. Ophthalmology 1999; 106: 1042-1043.

4 Lai JS, Lam DS, Tham CC. Limited instant laser peripheral iridoplasty in the management of acute primary angleclosure glaucoma. Eye 1999; 13: 26-30.

5 Lai JS, Tham CC, Chua JK, Lam DS. Immediate diode laser peripheral iridoplasty as treatment of acute attack of primary angle closure glaucoma: a preliminary study. J Glaucoma 2001; 10: 89-94.

6 Lam DS, Lai JS, Tham CC, Chua JK, Poon AS. Argon laser peripheral iridoplasty versus conventional systemic medical therapy in treatment of acute primary angle-closure glaucoma: a prospective, randomized, controlled trial. Ophthalmology 2002; 109: 1591-1596.

7 Sassani JW, Ritch R, McCormick S, Liebmann JM, Eagle Jr $\mathrm{RC}$, Lavery $\mathrm{K}$ et al. Histopathology of argon laser peripheral iridoplasty. Ophthalmic Surg 1993; 24: 740-745.

8 Lai JS, Tham CC, Chua JK, Poon AS. Laser peripheral iridoplasty as initial treatment of acute attack of primary angle-closure-long-term follow-up. J Glaucoma 2002; 11: 484-487.

9 Ritch R, Tham CC, Lam DS. Argon laser peripheral iridoplasty in the management of plateau iris syndrome: long-term results. Ophthalmology 2004; 111: 104-108. 